\title{
Dimensionamento estrutural da longarina de uma asa para competição SAE BRASIL Aerodesign: Comparativo do método analítico com o numérico.
}

\author{
Structural design of a wing spar for SAE Brazil Aerodesign competition: Comparative \\ between the analytical and numerical methods.
}

\author{
Marcelo de Paiva Bouçanova 1 (iD) https://orcid.org/0000-0001-5827-247X \\ Francisco Gilfran Alves Milfont, M.Sc. 1 (id) https://orcid.org/0000-0001-6934-3974 \\ ${ }^{1}$ Escola Politécnica de Pernambuco, Universidade de Pernambuco, Recife, Brasil. \\ E-mail do autor principal: paiva_marcelo94@outlook.com
}

\section{Resumo}

Este trabalho aborda o dimensionamento estrutural da longarina da asa de uma aeronave não tripulada para participar da competição SAE BRASIL Aerodesign, utilizando os métodos analítico e numérico de cálculo. Diversos perfis foram estudados para a construção da longarina, levando-se em conta as limitações geométricas do projeto e os materiais selecionados para a sua construção, a madeira balsa e o tubo de fibra de carbono, que foram ensaiados para se obter as suas propriedades mecânicas. A asa foi modelada utilizando o software SolidWorks ${ }^{\circledR}$ e para analise numérica foi utilizado o software ANSYS Workbench ${ }^{\circledR}$, que utiliza elementos finitos como base de cálculo. Os resultados obtidos, a partir da verificação das margens de erro entre os métodos, mostram que ambos podem ser utilizados para se obter um dimensionamento confiável da estrutura da longarina. Desta forma, este trabalho contribui para o conhecimento da aplicação da teoria do cálculo estrutural, e também para o aprimoramento de competências e habilidades nas áreas de engenharia mecânica e aeronáutica.

Palavras-Chave: Longarina da asa de uma aeronave; Análise estrutural; Competição SAE BRASIL Aerodesign.

\section{Abstract}

This work approaches the structural design of the wing spar of an unmanned aircraft to participate in the SAE BRASIL Aerodesign competition, using the analytical and numerical methods of calculation. Several profiles were studied for the construction of the stringer, taking into account the geometric limitations of the design and the materials selected for its construction, the balsa wood and the carbon fiber tube, which were tested to obtain their mechanical properties. The wing was modeled using SolidWorks ${ }^{\circledR}$ software and for numerical analysis the ANSYS Workbench ${ }^{\circledR}$ software was used, which uses finite elements as the basis of calculation. The results obtained, from the verification of the margins of error between the methods, show that both can be used to obtain a reliable stringer dimensioning. In this way, this work contributes to the knowledge of the application of structural calculus theory, as well as to the improvement of skills and abilities in mechanical and aeronautical engineering.

Key-words: Wing spar of an aircraft; Structural analysis; SAE BRAZIL Aerodesign competition. 


\section{Introdução}

Na análise estrutural de projetos de engenharia, um dos grandes problemas encontrados são as falhas ocorridas em seus componentes, devido aos esforços a que estes elementos são submetidos, o que pode comprometer toda sua integridade estrutural. Em projetos de aeronaves não é diferente e, para garantir segurança em voo e o desempenho desejado, é necessário que se faça a escolha correta do material a ser usado em suas estruturas e do seu correto dimensionamento para que se tenha um projeto confiável. Este trabalho apresenta alternativas para o dimensionamento estrutural da longarina da asa de uma aeronave não tripulada, que seja capaz de cumprir os requisitos da competição SAE Brasil Aerodesign, bem como estabelece uma metodologia confiável, que valide o dimensionamento da estrutura tanto com a utilização de métodos analíticos como numéricos, atendendo aos fatores de projeto adotados.

O desenvolvimento deste trabalho foi realizado a partir de estudos feitos a fim de determinar e calcular as cargas aerodinâmicas que atuam na asa da aeronave analisada, e então, analisar e escolher as dimensões de sua longarina principal, para que esta suporte tais esforços.

\section{Materiais e métodos}

A asa analisada neste trabalho foi a da aeronave que competiu na 19a Competição SAE BRASIL Aerodesign, representada pela equipe Tenpest Aerodesign em 2017. Ela foi modelada no software SolidWorks ${ }^{\circledR}$, versão 2016.

Para este trabalho foram utilizadas ferramentas computacionais para modelagem 3D, de cálculo e de simulação. A utilização destas ferramentas facilita a realização do comparativo entre os métodos de cálculo analítico e numérico, o que será de grande importância para a detecção das margens de erro entre os dois métodos abordados. Utilizou-se também uma máquina universal eletromecânica, micro processada, para ensaios mecânicos de tração e flexão, modelo EMIC Série 23-1MN, linha DL, existente na Universidade Federal de PernambucoUFPE, para se obter as propriedades mecânicas dos possíveis materiais que poderão compor a longarina, através da realização de ensaios destrutivos destes.
Para o método analítico, foram utilizadas as equações convencionais de resistência dos materiais, que fazem parte da área de conhecimento da engenharia mecânica. Para essa abordagem utilizouse como auxílio, os softwares Excel ${ }^{\circledR}$ e Mathcad ${ }^{\circledR}$, que facilitam a elaboração de um memorial de cálculo, acelerando o tempo do cálculo convencional, visto que este é um processo intensamente iterativo. Já para a análise numérica, utilizando elementos finitos, a asa foi modelada no software SolidWorks ${ }^{\circledR}$ e, posteriormente, analisada através do ANSYS Workbench ${ }^{\circledR}$, software que é muito utilizado em projetos de engenharia, por realizar simulações bastante confiáveis, principalmente quando se diz respeito a validações de critérios de falhas de projetos estruturais.

\subsection{Principais componentes estruturais da asa de um avião}

A estrutura da asa de uma aeronave é composta basicamente pelo bordo de ataque, bordo de fuga, longarinas principal e secundárias, ailerons, e perfis aerodinâmicos (nervuras), como pode ser visto na Figura 1.

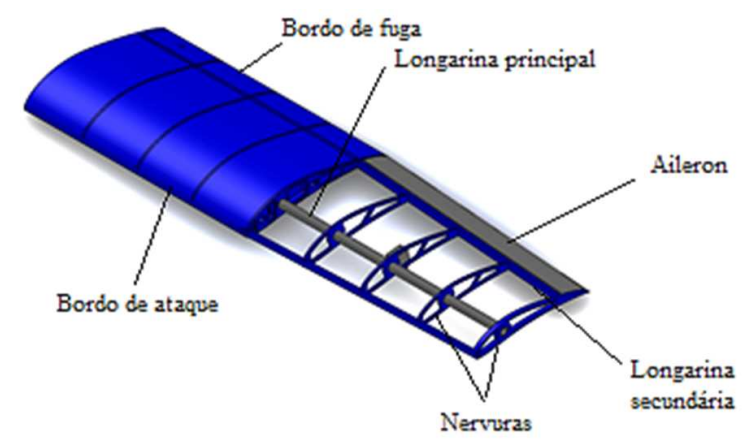

Figura 1: Modelagem da asa em estudo, com representação de seus principais componentes. Fonte: Autor.

A Figura 2 mostra uma vista superior do esqueleto da asa, com suas respectivas dimensões.

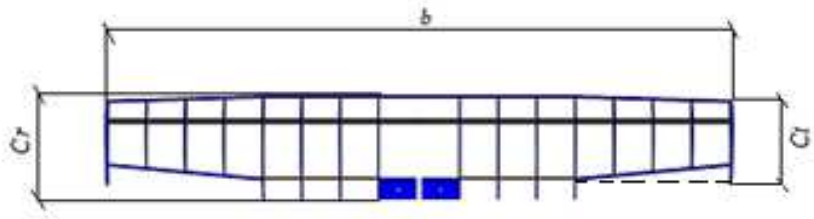

Figura 2: Asa que será analisada. Fonte: Autor. 
Onde, $b$ é a envergadura da asa; $C t$ é a corda da ponta da asa; e Cr é a corda na raiz da asa. A asa em estudo possui as seguintes dimensões: $b=1400 \mathrm{~mm}$, $C t=180 \mathrm{~mm}$ e $\mathrm{Cr}=200 \mathrm{~mm}$.

\subsection{Cargas atuantes na asa de uma aeronave}

Durante o voo, é gerada uma distribuição de sustentação ao longo da envergadura da asa que, segundo Anderson [1], atua no Centro de Pressão Aerodinâmico (CA) da asa, local onde esforços de flexão, torção e de cisalhamento se concentram, e também onde é posicionada a longarina principal da asa, objeto de estudo deste trabalho. Além disso, Rodrigues [3] menciona que o CA da asa localiza-se entre $25 \%$ e $30 \%$ da corda da asa, atrás do bordo de ataque da aeronave.

No caso em estudo, a função desta asa é de sustentar um peso total máximo de $44,14 \mathrm{~N}(4,5 \mathrm{kgf})$, que representa o peso da aeronave vazia somado ao peso da carga paga máxima (carga que será transportada em voo). Para calcular a distribuição de sustentação da asa de uma aeronave, é necessário se fazer a escolha do fator de carga $(n)$ deste avião. O fator de carga é definido como a relação entre a força de sustentação e o peso do avião. Um fator de carga, $n=1$, implica dizer que neste momento do voo, a sustentação é igual ao peso da aeronave, e esta está em equilíbrio, ou seja, sem alterar sua altitude em relação ao solo. Como durante um voo existem várias situações com fatores de carga variados, se faz necessário a escolha de um intervalo para o fator de carga, e este será escolhido seguindo a recomendação de Raymer [2]. Como as aeronaves da competição SAE BRASIL Aerodesign são aviões de pequeno porte, não tripulados, e que, quanto menor for o fator de carga, mais leve será a estrutura do avião, então, os fatores de carga escolhidos foram baseados na Tabela 1 .

\begin{tabular}{ccc}
\hline Categoria do avião & $n_{\text {pos }}$ & $\boldsymbol{n}_{\text {neg }}$ \\
\hline Pequeno porte & $2,5 \leq n \leq 3,8$ & $-1 \leq n \leq-1,5$ \\
Acrobático & 6 & -3 \\
Transporte civil & $3 \leq n \leq 4$ & $-1 \leq n \leq-2$ \\
Caças militares & $6,5 \leq n \leq 9$ & $-3 \leq n \leq-6$ \\
\hline
\end{tabular}

Tabela 1: Fatores de carga em função da categoria do avião. Fonte: Raymer (1922) [2].

Onde, $n_{\text {pos }}$ é o fator de carga positivo e $n_{\text {neg }}$ é o fator de carga negativo, que são usados de acordo com cada situação de voo.
Pelas características da aeronave, tomou-se:

$$
n_{\text {pos }}=2,5 \text { e } n_{\text {neg }}=-1
$$

O método escolhido para determinar a distribuição de sustentação da asa desta aeronave foi o Método da Aproximação de Schrenk, pois, segundo Rodrigues [3], este é um método simplificado que se aproxima bastante da distribuição real de sustentação na asa de aeronaves convencionais. Este método consiste em fazer a média aritmética entre a distribuição de sustentação de uma asa elíptica e a distribuição de sustentação da asa deste projeto, como mostram as equações:

$$
\begin{aligned}
& L(x)_{\text {Elíptica }}=\frac{4 L n}{b \pi} \sqrt{1-\left(\frac{2 x}{b}\right)^{2}} \\
& L(x)_{\text {Trapezoidal }}=\frac{2 L n}{b(1+\lambda)}\left[1+\left(\frac{2 x}{b}\right)(\lambda-1)\right] \\
& L(x)_{\text {Retangular }}=\frac{L n}{b} \\
& L_{n}=W n_{\text {pos }}
\end{aligned}
$$

Assim:

$$
L(x)_{\text {Schrenk }}=\frac{L(x)_{\text {Asa Mista }}+L(x)_{\text {Eliptica }}}{2}
$$

Onde:

$L(x)$ é a distribuição de sustentação ao longo da envergadura da asa para uma determinada geometria; $x$ é o ponto da envergadura que está sendo analisado; $L n$ é distribuição de sustentação com fator de carga; $W$ é o peso total da aeronave; $L(X)_{\text {Asa Mista }}$ é a distribuição de sustentação da asa em estudo; e $\lambda$ é a relação de afilamento da asa, que é dada pela equação (6).

$$
\lambda=\frac{C_{t}}{C_{r}}
$$

Com estes parâmetros definidos, e sabendo que a situação mais crítica é para o fator de carga positivo escolhido, foi possível gerar um gráfico no Excel ${ }^{\circledR}$, versão 2016, que representa a distribuição de sustentação de uma asa elíptica e da asa mista em estudo (asa composta por um trecho retangular e outro trapezoidal, como mostrado na Figura 2), e, a partir de suas médias, obteve-se a aproximação de Schrenk, ilustrada no gráfico da Figura 3.

http: / / dx.doi.org/10.25286/repa.v3i2.905 


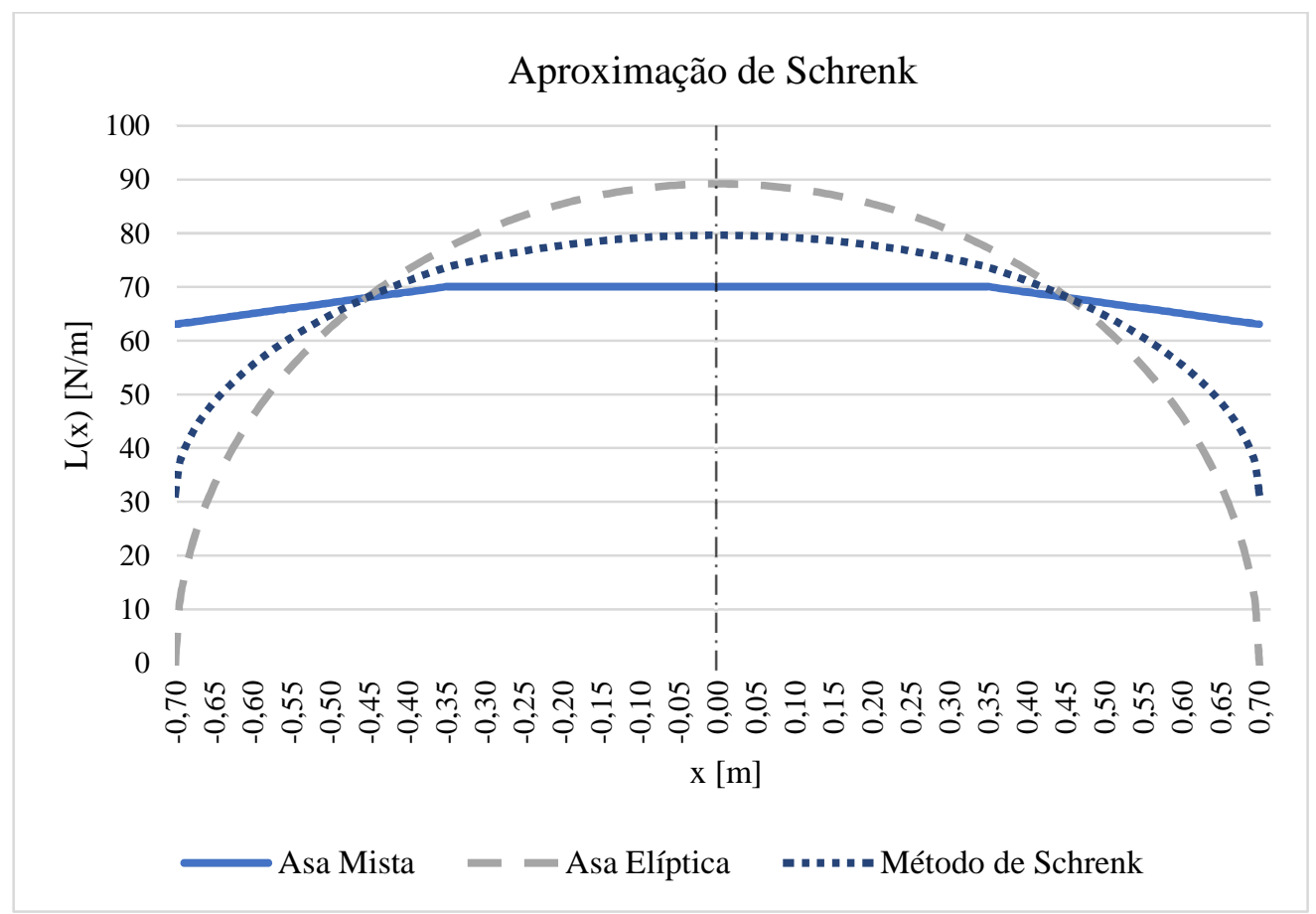

Figura 3: Gráfico da Aproximação de Schrenk na asa. Fonte: Autor.

A curva da aproximação de Schrenk pode ser representada pela seguinte equação polinomial de $6^{\circ}$ grau:

$$
\begin{aligned}
& L(x)_{S c h}=-1 * 10^{-9} x^{6}+6 * 10^{-7} x^{5}-8 * 10^{-5} x^{4}+5 \\
& * 10^{-3} x^{3}-61,537 x^{2}+0,212 x+79,69
\end{aligned}
$$

\subsection{Cálculo do esforço cortante e momento fletor na longarina através do método analítico}

Modelando a longarina da asa como uma viga engastada na fuselagem do avião, e tendo a equação que representa a curva de carga distribuída, pode-se determinar os valores de esforço cortante e momento fletor que atuam ao longo da longarina da asa, bem como seus valores máximos. Com auxílio do software Mathcad ${ }^{\circledR}$, versão 15.0 , utilizou-se o método das funções singulares, que torna possível a representação do esforço cortante e do momento fletor por simples expressões matemáticas. Elas são definidas, para $n \geq 0$, por:

$$
<x-a>^{n}=\left\{\begin{array}{cc}
(x-a)^{n} & \text { se } x>a \\
0 & \text { se } x \leq a
\end{array}\right\}
$$

$$
<x-a>^{0}=\left\{\begin{array}{ll}
1 & \text { se } x>a \\
0 & \text { se } x \leq a
\end{array}\right\}
$$

Onde, " $x$ " é o trecho de envergadura que será analisado e " $a$ " é onde se encontra o carregamento em análise.

Segundo Beer [4]:

$$
M(x)=E I \frac{d^{2} y}{d x^{2}}
$$

A partir da Equação (8), encontra-se a equação da declividade e da linha elástica da Iongarina, mostradas abaixo:

$\theta(x)=\frac{1}{E I} \int M(x) d x+c 1$

$y(x)=\frac{1}{E I} \int\left[\int M(x) d x+c 1\right] d x+c 2$

Onde $y(x)$ é a equação da linha elástica da viga, $M(x)$ é o momento fletor, $V(x)$ é o esforço cortante, $\theta(x)$ é a declividade da linha elástica, $E$ e $I$ são respectivamente 0 módulo de elasticidade e o momento de inércia da longarina, e $c_{1} c_{2}$ são constantes de integração.

As condições de contorno para a situação de 
engaste são:

- $\quad \operatorname{Para} x=0 \rightarrow y(x)=0$

- $\quad$ Para $x=0 \rightarrow \theta(x)=0$

Assim, obteve-se, através do método analítico, os diagramas de esforço cortante e momento fletor, representados nas Figuras 4 e 5, e os seus respectivos valores máximos:

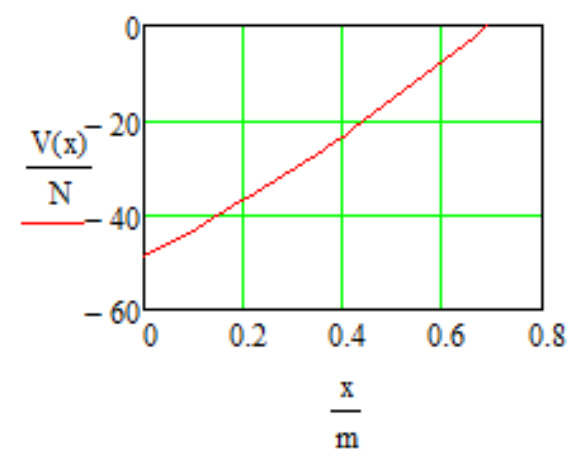

Figura 4: Diagrama de esforço cortante na asa. Fonte: Autor.

$$
V_{\text {máx }}=\left|V_{(0)}\right|=48,81 N
$$

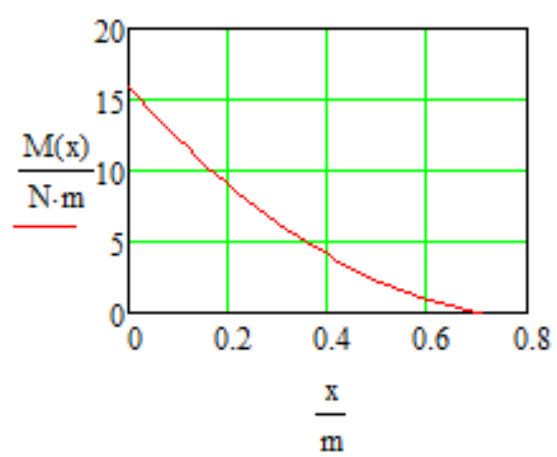

Figura 5: Diagrama do momento fletor na asa. Fonte: Autor.

$$
M_{\text {máx }}=15,88 \mathrm{Nm}
$$

\subsection{Escolha do perfil da longarina}

Para determinar o perfil da longarina, ou seja, o corte transversal deste componente, calculou-se inicialmente o momento de inércia mínimo necessário para que a estrutura suporte as cargas aerodinâmicas atuantes na asa. O momento de inércia mínimo pode ser obtido por meio da Equação (13).
Sabendo-se que:

$\sigma_{a d m}=\frac{M c}{I}$

E como,

$$
\sigma_{a d m}=\frac{\sigma_{y}}{C S}
$$

Tem-se que:

$$
I_{\text {mín }}=\frac{M_{\text {máx }} c}{\frac{\sigma_{y}}{C S}}
$$

Onde:

$\sigma_{a d m}$ é a tensão admissível, $\sigma_{y}$ é tensão de escoamento do material, CS o coeficiente de segurança e $I$ o momento de inércia da seção.

O coeficiente de segurança $(C S)$ escolhido foi baseado na norma CS-VLA [5], que sugere um $C S=$ 1,5 para estruturas primárias. Sabe-se que a tensão de escoamento $\left(\sigma_{y}\right)$ varia com o tipo de material a ser usado, e que a distância da linha neutra à extremidade do perfil (c), é limitada pela espessura da nervura no $C A$ da asa, que é de $30 \mathrm{~mm}$. Além disso, vale ressaltar que um perfil de longarina com base (largura) maior do que a altura implica em uma viga ineficiente, o que resultaria em aumento de peso desta estrutura. Portanto, será considerada a situação em que a base do perfil da longarina $(B)$ é igual a sua altura $(H)$. Assim, $B=H=20 \mathrm{~mm}(5 \mathrm{~mm}$ de folga na parte superior e inferior da nervura do perfil aerodinâmico, para permitir sua montagem). Logo, $c_{\text {máx }}=10 \mathrm{~mm}$.

Foram estudados os seguintes materiais para compor a longarina:

- Madeira balsa (densidade $=161 \mathrm{~kg} / \mathrm{m}^{3}$ ) com dimensões de $B=H=20 \mathrm{~mm}$ e espessura de chapas (abas e almas dos perfis) de $t$ $=6,3 \mathrm{~mm}$;

- Tubo de fibra de carbono (densidade = $\left.1565 \mathrm{~kg} / \mathrm{m}^{3}\right)$ com diâmetro externo $(D e)$ de $20 \mathrm{~mm}$ e interno $(\mathrm{Di})$ de $18 \mathrm{~mm}\left(c_{\text {máx }}=\right.$ $10 \mathrm{~mm})$; e De de $12 \mathrm{~mm}$ e $D i$ de $10 \mathrm{~mm}$ $\left(c_{\text {máx }}=6 \mathrm{~mm}\right)$.

A Tabela 2 mostra as dimensões $B, H$, De e Di e o comparativo dos diferentes perfis de Iongarina, utilizando os materiais acima.

http: / / dx.doi.org/10.25286/repa.v3i2.905 


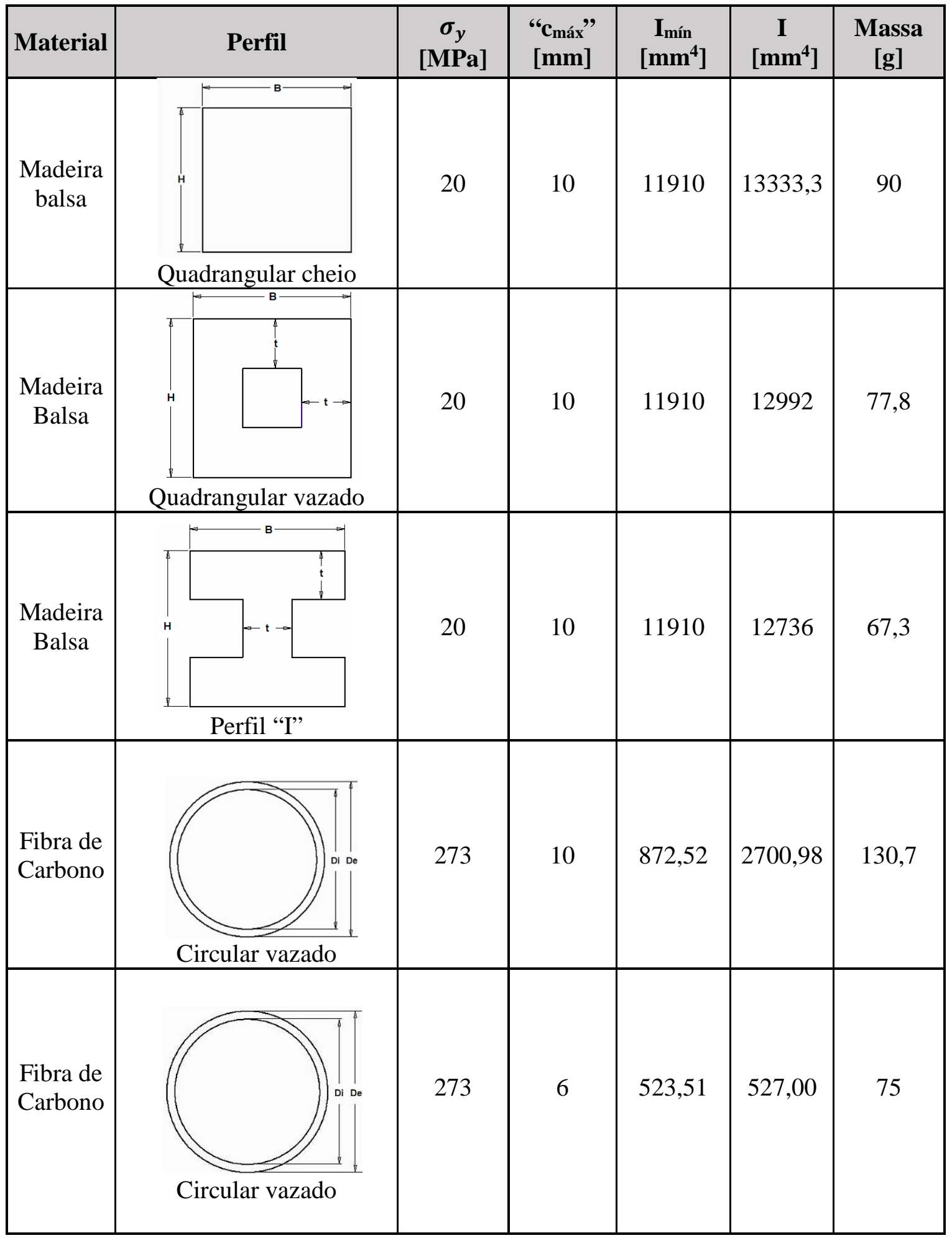

Tabela 2: Comparação de diferentes perfis de longarinas. Fonte: Autor. 
Os valores das tensões de escoamento mostradas na Tabela 2, foram obtidos por meio dos ensaios destrutivos destes materiais, segundo as recomendações das normas ASTM D. 790 [6] e ABNT. NBR-7190 [7]. As Figuras 6 e 7 ilustram os ensaios da fibra de carbono e da madeira balsa, respectivamente. As Figuras 8 e 9 mostram os gráficos de Tensão x Deformação, obtidos nos respectivos ensaios.

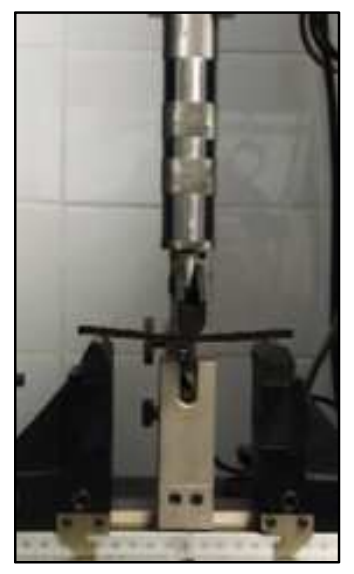

Figura 6: Ensaio destrutivo de flexão da fibra de carbono. Fonte: Autor.

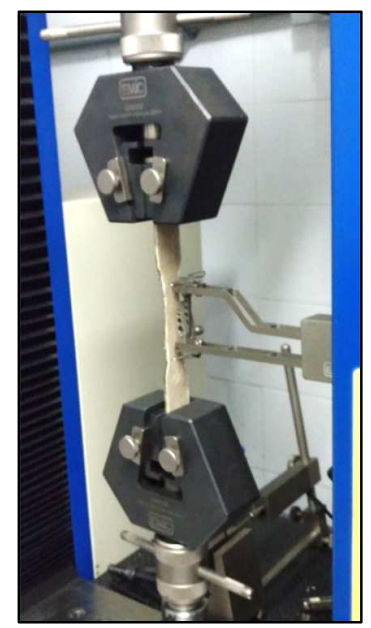

Figura 7: Ensaio destrutivo de tração da madeira balsa. Fonte: Autor.

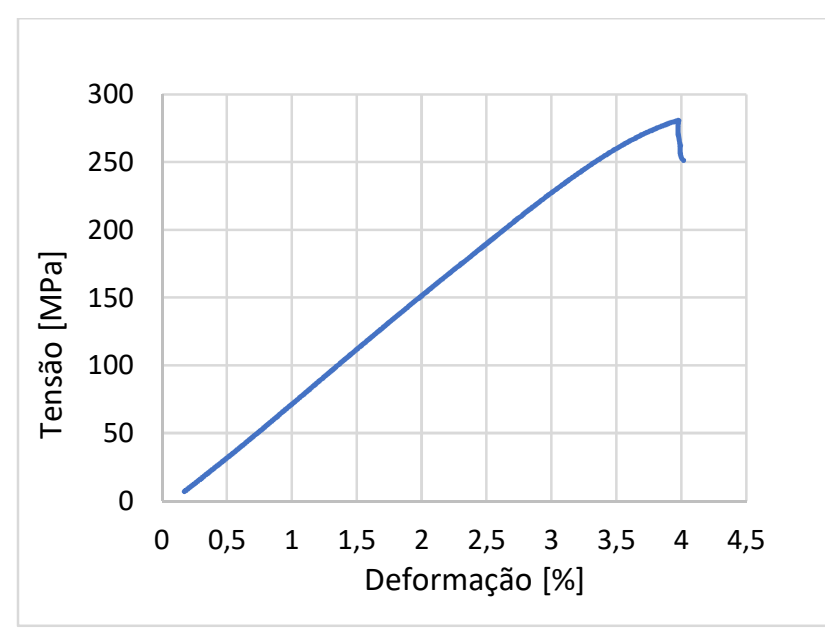

Figura 8: Gráfico de Tensão x Deformação obtido do ensaio destrutivo de flexão da fibra de carbono. Fonte: Autor.

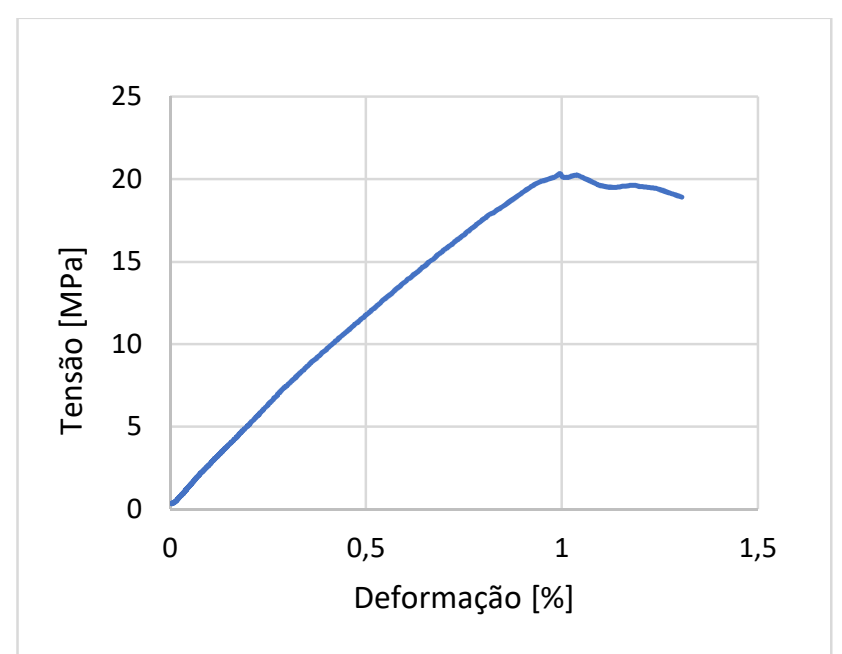

Figura 9: Gráfico de Tensão x Deformação obtido do ensaio destrutivo de tração da madeira balsa. Fonte: Autor.

Como pode se observar na Tabela 2, o momento de inércia do tubo de fibra de carbono com $20 \mathrm{~mm}$ de diâmetro externo e diâmetro interno de $18 \mathrm{~mm}$ é muito maior que momento de inércia mínimo necessário para que a estrutura suporte os esforços de voo. Portanto, para otimizar a estrutura e obter um momento de inércia mais próximo do mínimo, foi selecionado um tubo com diâmetro interno de $10 \mathrm{~mm}$ e externo de $12 \mathrm{~mm}$, o que implica em um $c=6 \mathrm{~mm}$ com um momento de inércia de $527 \mathrm{~mm}^{4}$, reduzindo a massa do tubo para $75 \mathrm{~g}$.

Assim, é possível concluir que o perfil circular vazado de fibra de carbono é mais vantajoso que os demais perfis, tanto na relação de Resistência/Peso, quanto em sua dimensão, pois a utilização de perfis com madeira balsa ultrapassaria a espessura útil 
dosperfis aerodinâmicos das pontas da asa $(24 \mathrm{~mm})$, comprometendo suas estruturas.

\subsection{Critério de falha através do método analítico}

O critério de falha adotado para o projeto da longarina foi o Critério da Máxima Energia de Distorção também conhecido como critério de von Mises.

Para a análise deste critério, é necessário calcular as tensões que atuam em cada eixo $(x, y, z)$ onde se encontra o elemento tridimensional em estudo, em seguida, determinar as tensões resultantes, e, por fim, verificar se o coeficiente de segurança encontrado satisfaz as condições recomendadas por [5].

A longarina engastada, com o carregamento de Schrenk, foi modelada no software Autodesk Inventor ${ }^{\circledR}$, versão 2016, e pode ser vista na Figura 10. A Figura 11, mostra em destaque o elemento tridimensional crítico, " $A$ ".

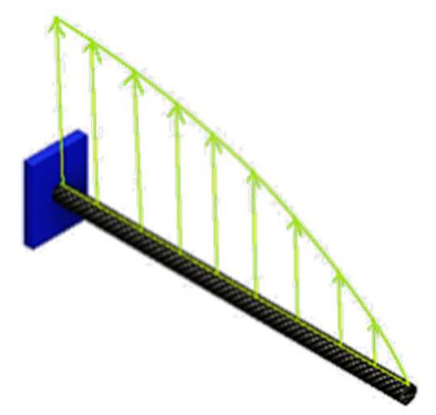

Figura 10: Metade da longarina engastada com carregamento de Schrenk. Fonte: Autor.

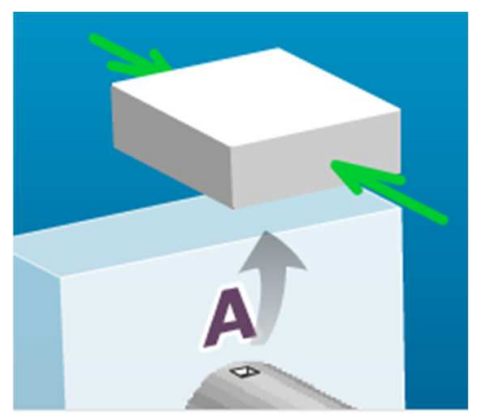

Figura 11: Elemento tridimensional crítico " $A$ " com os esforços de compressão que nele atuam. Fonte: Autor.
O momento fletor que atua na longarina é gerado pela distribuição de sustentação $L(x)$, e o ponto " $A$ ", que está sendo comprimido, foi o escolhido para ser analisado pois é considerado o mais crítico devido à disposição do carregamento, obtendo-se a tensão de flexão máxima:

$\sigma_{\text {flexão }}=\frac{M_{\text {máx }} c}{I}$

$$
\sigma_{\text {flexão }}=-180,80 \mathrm{MPa}
$$

Com estes dados, foi possível elaborar o círculo de Mohr, que está representado na Figura 12.

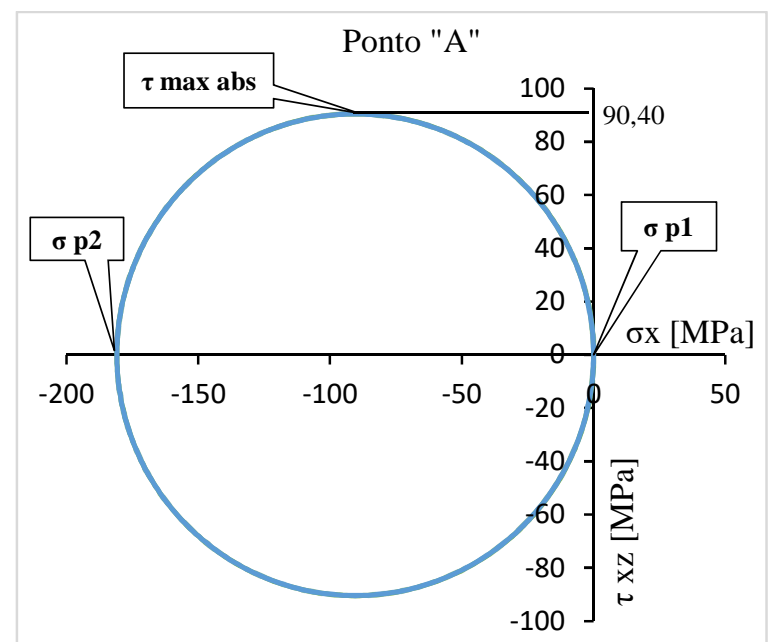

Figura 12: Círculo de Morh para o estado de tensão do ponto " $A$ ". Fonte: Autor.

Em que $\sigma_{p 1}$ e $\sigma_{p 2}$ são as tensões principais e $\tau_{\max a b s}$ é a tensão de cisalhamento máxima absoluta.

Por tratar-se de um estado plano de tensões, o coeficiente de segurança, pelo critério de von Mises, é expresso por:

$C S=\sqrt{\frac{\sigma_{Y}^{2}}{\sigma_{p 1}^{2}-\sigma_{p 1} \sigma_{p 2}+\sigma_{p 2}^{2}}}$

Encontrando-se para o ponto " $A$ ", submetido somente a tensão de compressão de $180,8 \mathrm{MPa}$, um $C S=1,51$ satisfazendo o fator de projeto recomendado pela CS-VLA [5]. 


\subsection{Cálculo do esforço cortante e momento fletor na longarina através do método numérico}

A análise numérica da longarina foi realizada utilizando-se o software ANSYS Workbench ${ }^{\circledR}$, versão 16.0, onde foram transferidos os valores da curva de
Tensão x Deformação obtida no ensaio da Figura 8. A longarina também foi modelada como uma viga engastada na extremidade em contato com a fuselagem e a aplicação do carregamento foi dividida em 7 componentes de forças, que visam simular a análise estática da curva de Schrenk atuante na Iongarina. A Figura 13 ilustra estas componentes, e seus valores, atuando ao longo da metade da longarina da asa.

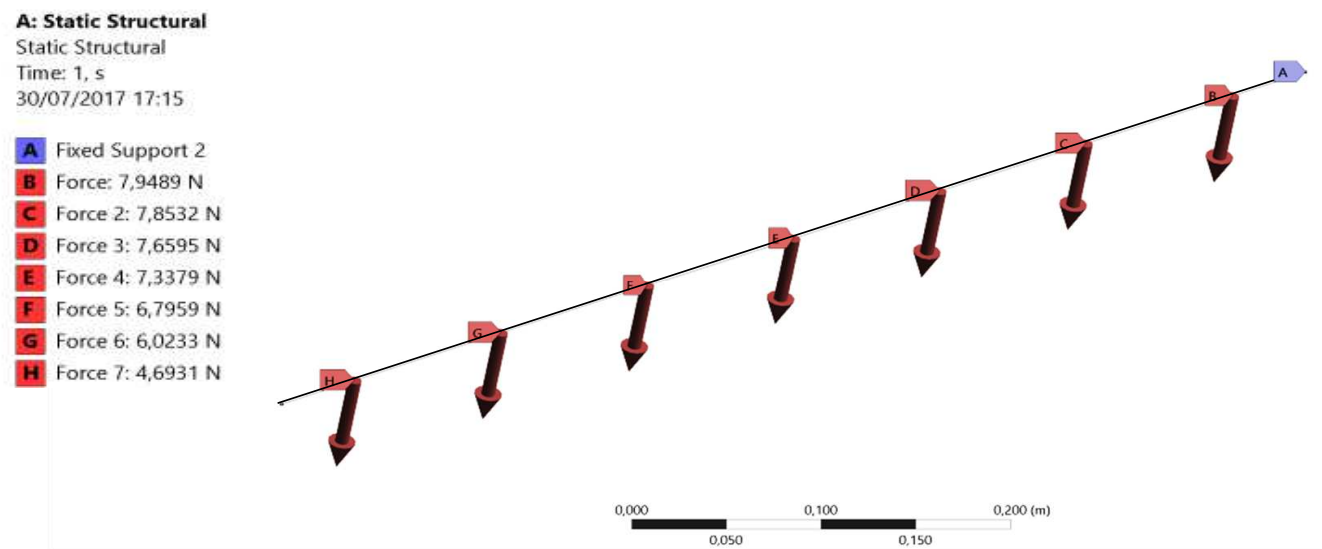

Figura 13: Modelagens de forças na longarina. Fonte: Autor.

Como o objetivo da simulação é obter o momento fletor e esforço cortante, para diminuir o tempo de processamento e sabendo que seriam obtidos valores concordantes com o cálculo analítico, foi utilizado o elemento finito de viga, obtendo-se, da simulação numérica, valores de esforço cortante e momento fletor de $48,31 \mathrm{~N}$, e $15,48 \mathrm{Nm}$ respectivamente. Com um erro comparado com o cálculo analítico de $-1 \%$ e $-2,5 \%$. As Figuras 14 e 15 mostram os valores obtidos para a simulação numérica.
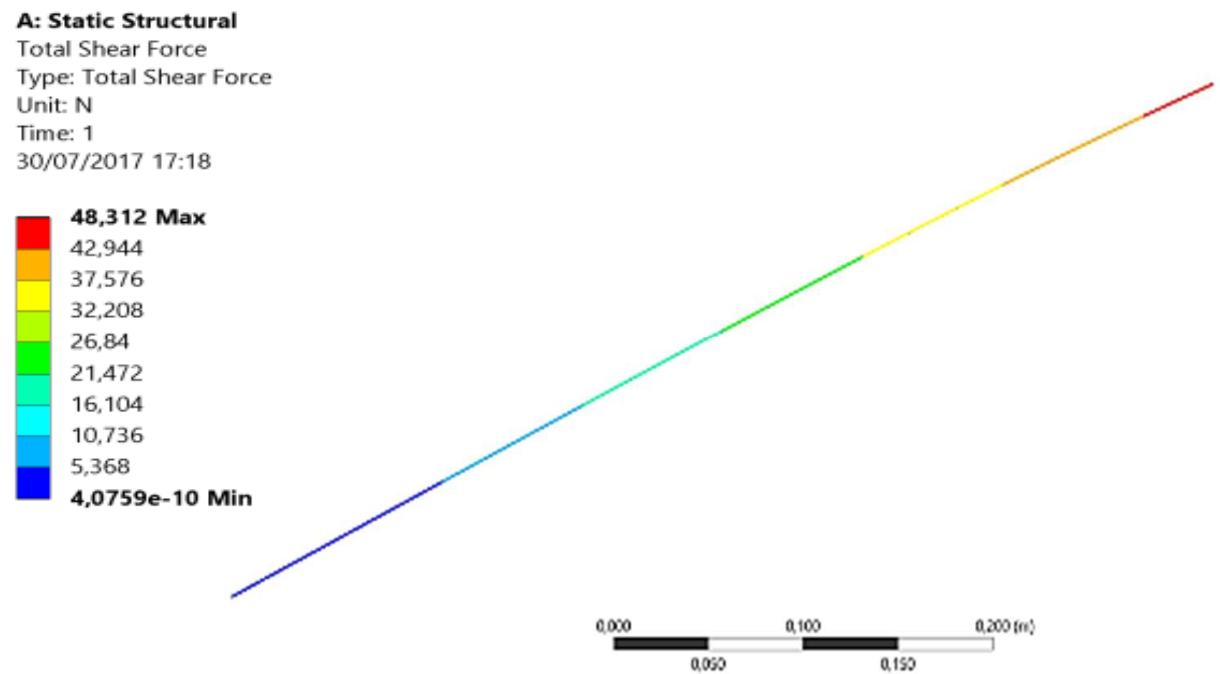

Figura 14: Esforço cortante na longarina utilizando o ANSYS Workbench ${ }^{\circledR}$. Fonte: Autor. 


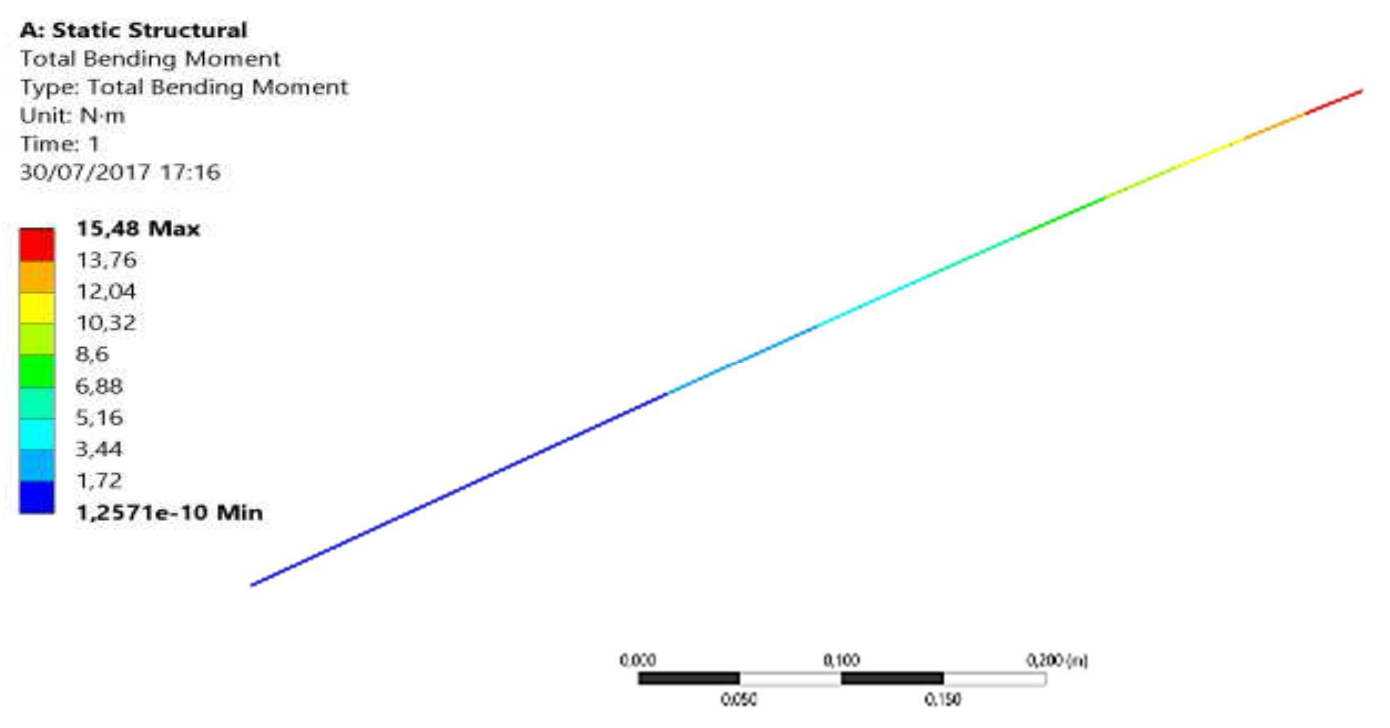

Figura 15: Momento fletor na longarina utilizando o ANSYS Workbench ${ }^{\circledR}$. Fonte: Autor.

\subsection{Critério de falha através do método numérico}

Na análise numérica, foi utilizado o mesmo critério de falha que se utilizou no método analítico, ou seja, o método da Máxima Energia de Distorção. Porém, para garantir confiabilidade da simulação, utilizou-se um Jacobiano (Jacobian Ratio) de 1,99 que indica que o mapeamento entre o espaço do elemento e espaço real está se tornando confiável computacionalmente. Foram usados 37.285 nós e 5.082 elementos do tipo tetraédrico parabólico na simulação, com Qualidade de Elemento (Element Quality) de 0,38, que representa uma satisfatória qualidade do refino da malha para esta análise. Pois, a partir de novos refinos, o resultado final da simulação praticamente não se altera. O coeficiente de segurança encontrado na simulação foi de 1,5. A Figura 16 ilustra a tensão equivalente de von Mises na asa.

\section{A: Static Structural}

Equivalent Stress

Type: Equivalent (von-Mises) Stress

Unit: $M P_{a}$

Time: 1

Q4/05/2018 10:35

\begin{tabular}{|l}
$183,47 \mathrm{Max}$ \\
163,09 \\
142,7 \\
122,32 \\
101,93 \\
81,544 \\
61,158 \\
40,772 \\
20,386 \\
$\mathbf{5 , 8 6 3 1 e - 6 ~ M i n}$
\end{tabular}
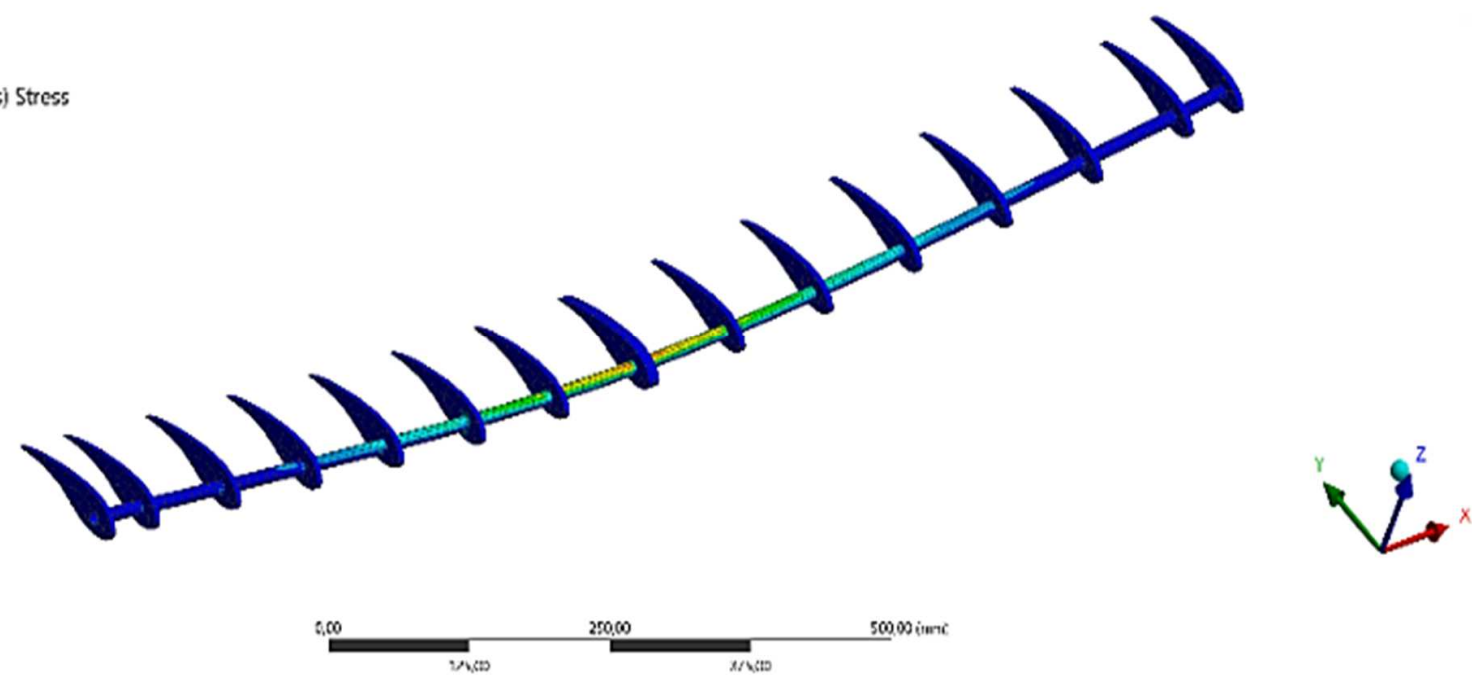

Figura 16: Tensão equivalente de von Mises. Fonte: Autor. 


\section{Resultados e discussões}

O trabalho resultou em uma metodologia confiável para o correto dimensionamento da longarina de uma aeronave não-tripulada, a partir do uso de elementos finitos, visto que as margens de erro são baixas quando comparadas ao método convencional. Estes erros podem ser reduzidos com o aumento do refino das malhas, porém isto levaria maior tempo de processamento computacional.

Além disso, o método numérico abordado neste trabalho torna-se uma ótima alternativa para o dimensionamento estrutural da longarina de uma asa, pois foi possível obter-se, de forma rápida e precisa, uma asa com alta resistência mecânica e de baixo peso, que está apta a cumprir os requisitos da competição nacional de Aerodesign, de forma competitiva.

Ainda se espera que este trabalho contribua para a evolução da equipe Tenpest tanto em questão a bonificação de relatórios técnicos na competição, como também para que ela possa desenvolver projetos estruturais mais confiáveis, e, consequentemente aviões estruturalmente mais competitivos.

\section{Conclusões}

A partir do comparativo feito entre os métodos de cálculo, é possível concluir que ambos podem ser utilizados para se obter um dimensionamento confiável da estrutura da longarina. Porém, o método numérico se mostra como uma alternativa mais rápida e eficaz, já que nele é possível se fazer análises estruturais com um nível de complexidade maior, que demandariam mais tempo através da utilização do método analítico. Além disso, esse tipo de método tem se difundido bastante no âmbito das engenharias mecânica e aeronáutica, o que se torna fundamental para o aprimoramento de competências e habilidades do aluno a nível de desenvolvimento pessoal e profissional.

\section{Referências}

[1] ANDERSON JR, J. D. Fundamentos de Engenharia Aeronáutica: introdução ao voo. São Paulo: McGraw-Hill, v. 200, p. 925, 2015.

[2] RAYMER, Daniel P. Aircraft design: A conceptual approach, american institute of aeronautics and astronautics. Inc., Reston, VA, p. 21, 1999.

[3] RODRIGUES, Luiz Eduardo Miranda José. Fundamentos da engenharia aeronáutica. Cengage learning, 2013.

[4] BEER, Ferdinand P. et al. Mecânica dos Materiais-7a Edição. AMGH Editora, 2015.

[5] CS-VLA, E. A. S. A. Certification Specifications for Very Light Aeroplanes, Amendment, 2009.

[6] ASTM, D. 790-03. Standard test methods for flexural properties of unreinforced and reinforced plastics and electrical insulating materials, v. 11, 2003.

[7] ABNT. NBR-7190-Projeto de estruturas de madeira. Rio de Janeiro, 1997. 